\title{
TTR
}

Traduction, terminologie, re?daction

\section{Traduction, rédaction, (franc)isation}

\section{Daniel Gouadec}

Volume 2, numéro 1, 1er semestre 1989

Carrefours de la traduction

URI : https://id.erudit.org/iderudit/037032ar

DOI : https://doi.org/10.7202/037032ar

Aller au sommaire du numéro

\section{Éditeur(s)}

Association canadienne de traductologie

ISSN

0835-8443 (imprimé)

1708-2188 (numérique)

Découvrir la revue

Citer cet article

Gouadec, D. (1989). Traduction, rédaction, (franc)isation. TTR, 2(1), 51-58.

https://doi.org/10.7202/037032ar d'utilisation que vous pouvez consulter en ligne.

https://apropos.erudit.org/fr/usagers/politique-dutilisation/ 


\section{Traduction, rédaction, (franc)isation}

\section{Daniel Gouadec}

Tout discours sur la traduction accorde implicitement ou explicitement à celle-ci un statut privilégié par rapport à la terminologie (terminographie?) elle-même surclassée par rapport à la rédaction: la traduction est considérée comme une activité intégrant la terminologie et de la rédaction.

Le «surclassement» idéologique de la traduction tient à plusieurs facteurs historiques (vision alchimiste-créatrice), techniques (technicité des textes traités; industrialisation des processus; recours à des aides matérielles et logicielles diverses), économiques (accroissement du chiffre d'affaires direct et des chiffres d'affaires induits), pédagogiques (promotion de la traduction au rang de disciplines d'enseignement), ou indirects (valorisation par le biais des enjeux de la terminologie «suscitée» par la traduction). Mais c'est surtout l'application d'un discours scientifique à la pratique et à la réflexion qui a fait de la traduction une entité indépendante.

La traduction ayant statut d'activité valorisée et valorisante, autonome et «intégrée», toute réflexion et tout discours la concernant ne peut que viser à renforcer son caractère d'autonomie en multipliant les objets et procédures ultra-spécifiques et en occupant au besoin un espace normalement réservé à des disciplines ou activités liées.

On ne s'étonne donc pas que, passé le stade des mesures d'urgence répondant à la nécessité de former "méthodiquement» des traducteurs dès l'instant où la demande dépassait l'offre «spontanée», le discours se soit «enrichi» par multiplication des objets et compartiments de la science et de la discipline d'enseignement.

Objet d'analyse fini, la traduction s'est refermée sur elle-même, phagocytant au passage la terminologie (les terminologies) et ce qu'il fallait de rédaction et de documentation pour lui garantir, précisément, son autonomie. Elle s'est surtout refermée sur la confrontation de séries d'objets linguistiques (textes de départ et textes d'arrivée) pour 
dresser, dans le cadre de l'analyse «scientifique», un inventaire des écarts culturels ou linguistiques types et proposer, dans le cadre "pédagogique», un ensemble aussi nettement défini que possible de moyens, procédés, ou procédures de construction de ponts culturels et/ou linguistiques entre univers et systèmes.

Quelque revendication théorique contraire que l'on puisse noter ici ou là, la traduction est inévitablement concernée par l'écart (écarttype ou écart atypique). Reste, à partir de cette évidence, une double question:

a) comment définir l'unité sur laquelle se fonde l'écart?

b) à quel moment doit-on prendre l'écart en compte?

La double question n'est pas innocente: la première touche à l'objet d'une éventuelle science; la seconde conditionne formation et pratique.

Dans la réalité courante, et bien que tout le monde s'accorde à reconnaître que langues, cultures et usages divergent d'un groupe à l'autre, le théoricien et le praticien s'appuient implicitement sur un parallélisme relatif entre formes linguistiques (notamment au niveau des structures d'énoncés) persillé d'écarts-types appelant des traitements ou des comportements spécifiques, notamment lorsqu'ils donnent lieu à apprentissage ou acquisition de réflexes. Le nombre d'unités d'évaluation est directement corrélé à la fréquence des écarts-types entre deux textes. On s'aperçoit ainsi que théoriciens, praticiens et pédagogues ont en commun de se laisser porter par la dérive de la «difficulté», du «piège», du "problème», bref, de l'écart-danger.

L'affinement des catégories de l'analyse et de l'enseignement provoque l'atomisation des unités considérées comme supports potentiels d'écarts. Toute traduction ou analyse de traduction étant soumise à équivalence quantitative et qualitative d'unités de traduction/unités d'évaluation, le nombre, la nature et la portée de ces unités conditionnent toute construction théorique et toute démarche pratique. Or, il faut savoir que le critère d' «équivalence" inter-linguistique/interculturelle change selon la taille de l'unité à l'intérieur de laquelle se calcule l'équivalence: totalité du document, chapitre, section, paragraphe, phrase, proposition, syntagme, unité terminologique ou lexicale. Donc, si l'on veut prendre en compte l'unité juste, il faut que celle-ci soit l'entité absolue du texte ou document résultant de l'activité de traduction. Par conséquent, il ne suffit plus de dire que toute traduction doit aussi engendrer un vrai texte ou document (cohérent et «lisible»); il faut considérer que toute traduction doit d'abord engendrer un vrai texte ou document (cohérent et naturel).

L'unité-texte doit avoir prééminence quelle que soit l'orientation de la traduction: traduction empruntant le schéma du texte premier 
ou traduction générant sa propre organisation et, parfois, certains de ces contenus.

Dans le premier cas, le traducteur s'appuie sur une structure proposée (imposée?) par le texte ou document premier et procède aux seules modifications imposées par les écarts-types. Les unités de traduction sont de taille réduite (syntagmes); le nombre, la nature et la distribution des objets et des thèmes dans le texte second sont identiques à ce qu'ils étaient dans le texte premier; les procédés d'écriture sont (sous réserve d'écarts-types) calqués sur ceux du texte premier.

Dans le second cas, la traduction est une sous-catégorie de la rédaction et obéit exactement aux mêmes principes et procédures que toute rédaction, à ceci près qu'elle tire une partie de ses déterminants de la représentation que construit le traducteur d'un texte pré-existant.

Entre ces deux pôles intervient une diversité de variantes combinant des traits empruntés à l'un ou l'autre type. Mais, toujours, les options se différencient selon la portée des unités fonctionnelles servant également d'unités de calcul d'équivalence: syntagme, proposition, phrase, ensemble de phrases, alinéa, paragraphe, ensemble de paragraphes, rubrique ou chapitre, etc. On comprend aisément que la traduction aboutant des syntagmes ne puisse s'apparenter à la traduction dont le seul critère d'équivalence s'applique à la globalité d'un message quoi qu'il advienne des constituants de celui-ci.

Nous posons deux postulats:

a) L'unité de traduction (production, évaluation) est l'entité texte ou document.

b) Cette unité est prospective en ce sens qu'elle intervient avant même que ne s'engage la traduction.

La mise en cuvre de cette unité est tributaire, dans l'exemple de la traduction vers le français, d'un projet de francisation dont le degré peut varier: francisation absolue ou francisations partielles des contenus et des caractères d'un document (chronologiquement) premier.

La francisation des contenus relève principalement mais non exclusivement du transfert ou de l'adaptation culturel(le) / expérientiel(le); la francisation des caractères du document premier relève principalement mais non exclusivement du transfert ou de l'adaptation rhétorique/rédactionnelle et linguistique. En pratique, les deux faces du transfert ou de l'adaptation présentent de multiples points d'intersection.

Si l'on pousse au-delà de la francisation pour étendre le champ de la traduction à l'adaptation du document second (résultat de l'activité de traduction) à ses destinations (public spécifique et mode d'utilisation spécifique du document), la traduction tend vers la rédaction (produc- 
tion) "pure» d'un texte ou para-texte dont les degrés de convergence typologique, formelle, structurelle avec un texte ou document premier se réduisent ${ }^{1}$.

À n'en pas douter, tout praticien ou théoricien est profondément convaincu que «traduction = (franc)isation + rédaction» et que l'on ressuscite ici de vieilles lunes. Dont acte. Mais, à y regarder de plus près, est-on certain que la pratique ne contredise pas la déclaration d'intention qui ne serait alors qu'alibi? Est-on certain que tous les corollaires des postulats auxquels on affirme souscrire depuis si longtemps sont effectivement pris en compte dans la pratique et dans la construction théorique?

La francisation absolue est la reformulation de la résultante synthétique d'un ensemble de thèmes portant sur des objets particuliers et encadrés par des circonstants spécifiques: un thème générique inscrit dans la reconstitution du projet ayant guidé la production du document premier. Elle prend appui, dans la synthèse interprétative, sur une unité-document globalisée et non sur des empilages de sous-unités. Elle s'organise, dans la production du texte ou document second, en fonction d'un projet global emboîtant, dans l'ordre, les cadres génériques (lieu, langue, moment, domaine, secteur), les cadres de destinations (nature du public et fonctions du document) et les cadres typologiques (type du document second) qui, ensemble, conditionnent tous les choix ultérieurs.

Lorsque la francisation est "relative» ou partielle, le projet fait explicitement référence à ses limites ${ }^{2}$.

Le projet de traduction fixe d'emblée l'écart générique entre document premier (analysé) et document second (en projet). Il préempte ainsi tous les écarts spécifiques qui sont encore, à ce stade, largement imprévisibles, puisqu'ils découlent du premier (degré d')écart générique. Il détermine aussi, pour une large part, le trajet prospectif du texte ou document second.

1. Le type auquel appartiendra le texte ou document second peut être fondamentalement différent de celui auquel appartenait le texte premier. Ceci est particulièrement vrai si l'on doit, par choix ou par obligation, adapter la traduction à des modalités particulières de communication. Ainsi se définissent des types spécifiques de traductions. Voir «Autrement dire» (article à paraître dans Meta).

2. Les limites de la francisation peuvent concerner la rémanence de certaines formes de l'original (comme, par exemple, dans le cas de non-francisation de messages s'affichant sur l'écran de tel matériel) ou le blocage des unités d'équivalence dans des situations telles que la traduction "page-à-page» ou «alinéa pour alinéa» ou encore «ligne pour ligne» imposées par le donneur d'ouvrage ou revendiquées par le traducteur. Ceci conditionne de toute évidence les éléments du projet de document ou texte second. 
Le trajet du document ou texte second fait se succéder les diverses rubriques ou sections de traitement dont les cadres de second niveau déterminent les objets, la nature des thèmes et leur organisation. Chaque sous-unité correspondant à une rubrique ou à une section de traitement repose sur la conjonction d'un projet et d'un trajet. Ainsi, telle section de traitement de contenus peut correspondre à un projet de définition ou de directive ou de stipulation d'interdiction et se mettre en place selon un trajet spécifique: exiger le choix de tel objet ou de tel thème, solliciter tel enchainement des thèmes, etc.

L'ensemble des marqueurs du trajet de production-construction du document ou texte second correspond à une sorte de guide analytique ou "bande de déroulement" comportant, pour chaque rubrique ou section:

* son type et ses finalités

* ses cadres (imbriqués dans les cadres génériques)

* son objet propre et son thème générique.

On retrouve à ce niveau, l'enchaînement des préemptions du projet global. Le degré de ramification du trajet dépendra de la nature et de la taille des unités sur lesquelles repose implicitement ou explicitement le calcul des équivalences (critères de qualité de traduction).

La production ou rédaction effective du document ou texte second développe le thème générique et le thème de chaque rubrique ou section en ramifiant les circonstants, les objets et les sous-thèmes. Elle repose aussi, bien entendu, sur le choix des vecteurs linguistiques et des enchainements (distribution et nature des facteurs de cohésion et de cohérence) les mieux adaptés à la teneur du message à transmettre, aux exigences de la rhétorique et, inévitablement, au projet de calcul d'équivalences (nature et taille des unités constitutives du document).

L'efficacité de communication commande d'utiliser toute accroche nécessaire pour «mobiliser» le lecteur, de respecter toute convention de présentation imposée ou justifiée, d'exclure tout élément susceptible de bloquer la lecture ou tout élément de contenu ou de forme superflu ou inutilement redondant, de respecter toute stéréotypie de contenu ou de forme imposée par la nature du projet, de respecter les principes de «logique universelle» et les règles de la grammaire «technique" concernée, de baliser clairement le trajet de la lecture, de respecter toutes les conventions régissant la nature et l'enchaînement des rubriques ou sections du type de texte ou de document retenu, de respecter toutes les conventions régissant les stéréotypies de formulation-organisation à l'intérieur du type de texte ou de document retenu, de prendre en compte les mécanismes interprétatifs mis en œuvre, ou susceptibles de l'être, par le lecteur ${ }^{3}$.

3. Lorsque les mécanismes interprétatifs ne peuvent plus jouer, le lecteur comprend «littéralement" le segment de texte concerné mais ne peut plus établir, par présupposés et implications, sa fonction dans le texte. Il y a donc incongruité. 
La progression ci-dessus décrit, très précisément, la rédaction. Elle correspond tout aussi précisément à ce que devrait être la traduction lorsqu'elle ne repose pas sur le calque rompu par les seuls écarts-types dans une forme de traduction appelant systématiquement l'équivalence quantitative des syntagmes. Nous pensons en effet que le traducteur devrait, dans la mesure du possible, exploiter des emboittements de stéréotypes en ne faisant référence au texte premier que pour y prendre les variables à intégrer aux stéréotypes aux différents niveaux auxquels ils interviennent pour pré-construire des éléments du document ou texte second.

En fait, le seul véritable point de rencontre systématique entre le texte premier et le texte second est, quelles que soient les conditions particulières de la traduction, la combinaison «thème générique + destinations" - avec, le cas échéant, substitution de l'objet imposée par une divergence culturelle fondamentale. Sur la convergence thématique et fonctionnelle peuvent se greffer divergences ou convergences de types de documents, de nature, de nombre et d'enchaînement de rubriques, etc. Le nombre et le niveau des convergences ou divergences conditionnent les écarts constatés a posteriori entre texte premier et texte ou para-texte second sauf, rappelons-le, lorsque la multiplication des points de convergence entre unités sub-textuelles constitue une obligation imposée par le donneur d'ouvrage ou par les circonstances (documents traduits alinéa par alinéa afin de faciliter les mises à jours ultérieures) ou un choix délibéré du traducteur (traduction juxtalinéaire indiquée) ou une sorte de "garantie" pour le traducteur qui, par insuffisance de maîtrise, cherche refuge dans le calque des unités syntaxiques ou phrastiques.

Et c'est précisément dans ce dernier cas qu'il faudrait que le projet textuel global renforçât son emprise sur l'ensemble des atomes constitutifs du texte final.

La différence est différence de points d'appui. Dans le premier cas, l'ensemble «projet + guide analytique» forme point d'appui globalisé; dans le second, les points d'appui se multiplient à mesure que se réduisent en portée les unités de transfert, MAIS un projet global cohérent (unificateur) est alors plus nécessaire que jamais. En d'autres termes, la référence à des critères de rédaction devrait se renforcer à mesure que le fractionnement des sous-unités augmente la difficulté de maintenir cohérence et cohésion. Il paraît invraisemblable que l'on puisse envisager de traduire sans avoir formé un projet global gouvernant le processus alors que l'on n'envisagerait guère de rédiger sans maîtrise préalable de tous les tenants et aboutissants de l'activité de rédaction.

La référence à un ensemble "projet + guide analytique» est, de toute évidence, toujours nécessaire si l'on considère que rares sont les 
traducteurs absolument maîtres des systèmes d'anaphore-cataphore, des modalités d'intégration des circonstants et des distributions de composants dans leurs unités (propositions, phrases, paragraphes, sections) supra-ordinales. Il suffit, pour s'en convaincre, d'analyser les écarts de «style» chez un même individu selon qu'il rédige librement ou qu'il traduit ou de relever dans les textes traduits des types spécifiques, récurrents, de caractères que l'on ne retrouve pas dans les textes rédigés.

On note, en particulier, une rupture plus ou moins marquée des stéréotypies à chacun des niveaux du document ou texte second. Cette rupture des stéréotypies constitue la plus sérieuse des menaces d'abâtardissement des textes lorsqu'il y a importation directe de stéréotypes étrangers mais aussi lorsque les stéréotypes naturels font défaut. La traduction exigerait, aux fins de francisation minimale, le recours aux stéréotypies culturelles, linguistiques, rhétoriques au niveau de la structure générale des textes ou chapitres ou sections (mode d'emploi, note de service, rapport, lettre, etc., présentant les caractères naturels du type en français), au niveau de chaque mode d'articulation rhétorique (démonstration mathématique, explication, définition, etc., présentée selon une matrice logico-linguistique naturelle en français), au niveau des unités phraséologiques standard ou formules (exemple, restriction, relation de cause à effet, etc., introduits par l'une des formules naturelles possibles), au niveau des unités phraséologiques spécifiques (syntagmes ou expressions «formulés» dans le respect des jargons du groupe concerné).

Il est probable que seule la généralisation de l'effet de stéréotypie permet de NATURALISER les éléments constitutifs du texte ou document second et donc de prévenir les effets négatifs de traductions asservies générant l'abâtardissement linguistique-rhétorique-culturel. Il est certain que les enchâssements de stéréotypes doivent s'organiser du général au particulier selon le guide posé par la dyade «projet + trajet». Ils commencent avec le choix d'une matrice textuelle naturelle, continuent avec les choix de matrices de rubriques ou de sections naturelles, puis avec les choix d'enchaînements naturels, touchent les «formules» propositionnelles de la combinatoire syntaxique puis mobilisent les stéréotypies lexicales déjà recensées sous le nom de terminologies. En d'autres termes, il faut étendre à toute unité supra-ordinale, et jusqu'à la totalité du texte, les principes appliqués aux stéréotypes terminologiques.

Il faudra bien qu'un jour le traducteur exploite sciemment des modèles génériques (discours non spécialisés) ou spécifiques (discours spécialisés) originaires de sa langue-culture-rhétorique et qu'il les impose à ce qu'il est censé naturaliser. Il aura, ce faisant, étendu la notion d'écart-type (stéréotype) à l'intégralité du texte et mis en place d'emblée une structure dans laquelle viendront se ranger toutes les 
formes de stéréotypie de moindre portée. Il aura aussi simplifié considérablement sa tâche dans la mesure où il ne lui appartiendra plus de "fabriquer" ou "calquer» avec plus ou moins de bonheur ce que d'autres ont déjà, sans arrière-pensée d'aucune sorte, pré-construit. Peut-être est-il temps de constituer une sorte de trésor phraséologique et typologique (une banque de stéréotypes) intéressant les textes généraux et les textes de spécialité comme chaque section «logique / rhétorique»? Ce trésor phraséologique et typologique constituerait à n'en pas douter une aide précieuse à la rédaction et à la traduction ou, à un niveau plus général, à la francisation ${ }^{4}$.

Il peut sembler paradoxal que ce qui commence comme un plaidoyer pour la rédaction se termine comme un appel à l'aboutage d'éléments préfabriqués entre lesquels il suffirait de remplir des blancs. Ce n'est vrai que si l'on conserve de la rédaction une image de création alchimique et il va de soi que les textes créatifs continueront de faire appel à une traduction elle-même créative. Mais pourquoi faudrait-il voir un appauvrissement dans l'application des stéréotypies au gruau récursif, redondant, déstructuré, que moulinent inlassablement les traducteurs? Dans le contexte de traduction vers le français que nous avons retenu, la langue et la culture d'accueil auraient tout à gagner de la francisation absolue des formes et des contenus, même (ou peut-être surtout) si la stéréotypie générale finit par faire émerger une structure unique par type conduisant au blocage définitif des choix. En pareil cas, on renverrait à la machine ce qui serait ainsi devenu mécanique et on ne soumettrait au traducteur humain que les documents sollicitant l'intelligence naturelle: documents "riches" et formes de traduction requérant, du fait d'une modification marquée des circonstants de la communication et donc d'un écart marqué entre projet du texte premier (achevé) et projet de production du texte ou para-texte second (à venir), une très forte dose d'analyse / extraction / synthèse / recomposition / réécriture.

Université de Rennes II

4. Espérons simplement que l'éventuel traitement des unités typologiques et phraséologiques ne suscite ni phraséographie ni phraséotique ni autres boursouflures verbales. 